from baseline to Week 12 (FP/FORM: +0.196 L; FP/SAL: +0.257 L). The LS mean difference in change in pre-dose $\mathrm{FEV}_{1}$ was $-0.061 \mathrm{~L}$ between treatments ( $95 \%$ CI -0.161 to 0.040 ). Non-inferiority of FP/FORM to FP/SAL was demonstrated (the lower limit of the $95 \%$ CI exceeded the acceptance limit of $-0.2 \mathrm{~L})$. In total, $72.3 \%(73 / 101)$ patients started on FP/FORM 250/10 $\mu$ g and $75.2 \%(76 / 101)$ on FP/ SAL $250 / 50 \mu$ g. Eight patients (FP/FORM: $n=5$; FP/SAL: $n=3$ ) required an increase in dose. Similar numbers of patients treated with FP/FORM and FP/SAL discontinued due to lack of efficacy (FP/ FORM: $\mathrm{n}=1$; FP/SAL: $\mathrm{n}=2$ ). Twenty-three patients $(11.4 \%)$ experienced mild or moderate asthma exacerbations. Four patients $(2.0 \%)$ experienced severe exacerbations (FP/FORM: $n=3$; FP/SAL: $n=1$; $\mathrm{p}=0.621$ ). Overall, $23.8 \%$ of patients experienced at least one AE. The rate was the same in both treatment groups (24/101). Most AEs were mild or moderate. No clinically important changes in laboratory results, vital signs or ECGs were observed.

Conclusion FP/FORM and FP/SAL had similar efficacy and safety profiles.

Abstract P177 Table 1 Pre-dose FEV 1 at Week 12-per protocol set

\begin{tabular}{|c|c|c|c|c|c|c|}
\hline & \multirow[b]{3}{*}{$\mathbf{n}$} & \multicolumn{5}{|c|}{ Pre-dose FEV $_{1}$ (L) } \\
\hline & & \multicolumn{2}{|l|}{ Week 12} & \multicolumn{3}{|c|}{ Difference FP/FORM - FP/SAL } \\
\hline & & LS mean & $95 \% \mathrm{CI}$ & LS mean & $95 \% \mathrm{CI}$ & $P$ value \\
\hline FP/FORM & 96 & 2.402 & 2.324 to 2.481 & -0.061 & -0.161 to 0.040 & 0.007 \\
\hline $\mathrm{FP} / \mathrm{SAL}$ & 95 & 2.463 & 2.384 to 2.543 & & & \\
\hline
\end{tabular}

\section{P178 VALIDATION OF A NOVEL SYNTHETIC ABSORPTIVE MATRIX (SAM) FOR SAMPLING NASAL MUCOSAL LINING FLUID}

doi:10.1136/thx.2010.151043.29

${ }^{1} \mathrm{D}$ Jackson, ${ }^{2} \mathrm{Y}$ Clements, ${ }^{1} \mathrm{~S} \mathrm{~L}$ Johnston, ${ }^{1} \mathrm{~T} \mathrm{~T}$ Hansel. ${ }^{1}$ Imperial College London, London, UK; ${ }^{2}$ Meso Scale Discovery, London, UK

Introduction An improved method for sampling nasal mucosal lining fluid (MLF), termed nasosorption, utilises a synthetic absorptive matrix (SAM) (Accuwick Ultra, Pall). Conventionally, Whatman's filter paper has been used for absorption of nasal MLF, but this natural cellulose source has the capacity to bind mediators, causing eluted fluid to have decreased and variable detectable levels of mediators. Nasal lavage has the problem of diluting MLF and this also causes detectable levels of mediators to be low. Strips of Accuwick are effective for nasosorption in adults following nasal allergen challenge and for sampling children with active rhinitis. However, Accuwick is no longer manufactured and we wished to validate an alternative SAM (Leukosorb, Pall).

Methods Sputum supernatant $(40 \mu \mathrm{l})$ from a subject with COPD as well as a standard preparation of cytokines was spiked onto Accuwick and Leukosorb strips. Following elution by spin filter centrifugation, the MesoScale Diagnostics (MSD) multi-immunoassay platform was used to assess levels of IFN- $\gamma$, IL-10, IL-12 p70, IL-6, IL-8, and TNF $\alpha$. After absorption to Accuwick and Leukosorb, elution was compared with and without buffer (PBS with BSA (1\%) and Triton $\mathrm{X}(1 \%)$ ), prior to immunoassay of the recovered sample. Results Without buffer the recovery of 7 cytokines after the sputum supernatant was applied to Accuwick was a mean of $17.6 \%$ (range $1-100 \%$ ), while recovery was a mean of $20.2 \%$ (range $2.9-92.5 \%$ ) using Leukosorb. Addition of the buffer prior to elution of the fluid increased mean recovery to $61.8 \%$ when employing the Leukosorb system. Finally, in a direct comparison when employing Leukosorb and Accuwik for nasosorption in different nostrils after nasal allergen challenge in a single subject, Leukosorb resulted in higher detectable IL-5 levels in MLF.

Conclusion Leukosorb appears to be a superior alternative to Accuwick Ultra for nasosorption in terms of recovery of cytokines. Addi- tion of a buffer containing detergent and protein prior to elution significantly increases recovery. SAM has the potential to be employed in the upper and lower respiratory tract to sample undiluted MLF.

\section{P179 LUNG-BASED ASSESSMENT IN ANDERSON FABRY DISEASE (AFD) DEMONSTRATES DIFFERENTIAL $\alpha$ GALACTOSIDASE A ENZYME (GLA) ACTIVITY AT BLOOD AND TISSUE SITES}

doi:10.1136/thx.2010.151043.30

${ }^{1} \mathrm{~N}$ T Shafi, ${ }^{1} \mathrm{D}$ A Hughes, ${ }^{2} \mathrm{R}$ Baker, ${ }^{3} \mathrm{C}$ Smith, ${ }^{1} \mathrm{~J}$ Hurst, ${ }^{2} \mathrm{~A}$ Mehta, ${ }^{1} \mathrm{M}$ Lipman. ${ }^{1}$ UCL Medical School/Royal Free Hospital, London, UK; ${ }^{2}$ Royal Free Hospital, London, UK ${ }^{3}$ UCL Medical School, London, UK

Introduction and Objectives AFD is an X-linked lysosomal storage disorder caused by mutations of the GLA gene. Lack of enzyme results in storage material accumulation within lysosomes, leading to multi-organ pathology. Airflow obstruction has been reported, though there has been no systematic assessment of lung enzyme activity in affected individuals. We therefore undertook a controlled cohort review of UK AFD patients focussing in particular on the relationship between airway and blood intra- and extracellular GLA, and lung pathology.

Methods Study subjects and controls were recruited following local Ethics Committee approval. All underwent systematic pulmonary investigation, including lung function testing and sputum induction with $40 \mathrm{ml}$ of $4 \%$ hypertonic saline over $20 \mathrm{~min}$ via an ultrasonic nebuliser. GLA activity was measured from AFD patients and healthy controls in induced sputum (IS) cells and supernatant using a fluorometric assay. In addition, in AFD patients, GLA activity was measured simultaneously in blood leucocytes and plasma.

Results 45 AFD patients with variable severity extra-pulmonary disease were recruited ( 20 males, 10 smokers). The population had a mean FEV1 of $89 \%$ predicted. 20 of 45 (44\%) (13 males, 6 smokers) had evidence of airflow obstruction with FEV1/FVC ratio $<70 \%$. IS intra- and extracellular GLA activity was lower in AFD affected males compared to controls ( $\mathrm{n}=18$ and 13 , median enzyme activity $18.8 \mathrm{vs} 41.7 \mathrm{nmol} / \mathrm{h} / \mathrm{mg}$ protein and $0.9 \mathrm{vs} 10.0 \mathrm{nmol} / \mathrm{h} / \mathrm{ml}, \mathrm{p}<0.001$ and $p<0.01$, respectively). No similar difference was found in females. Paired blood and sputum data from 22 AFD patients (13 males, 18 on enzyme replacement therapy) demonstrated greater

Comparison of paired blood and induced sputum leucocyte enzyme activity in AFD males and fem ales

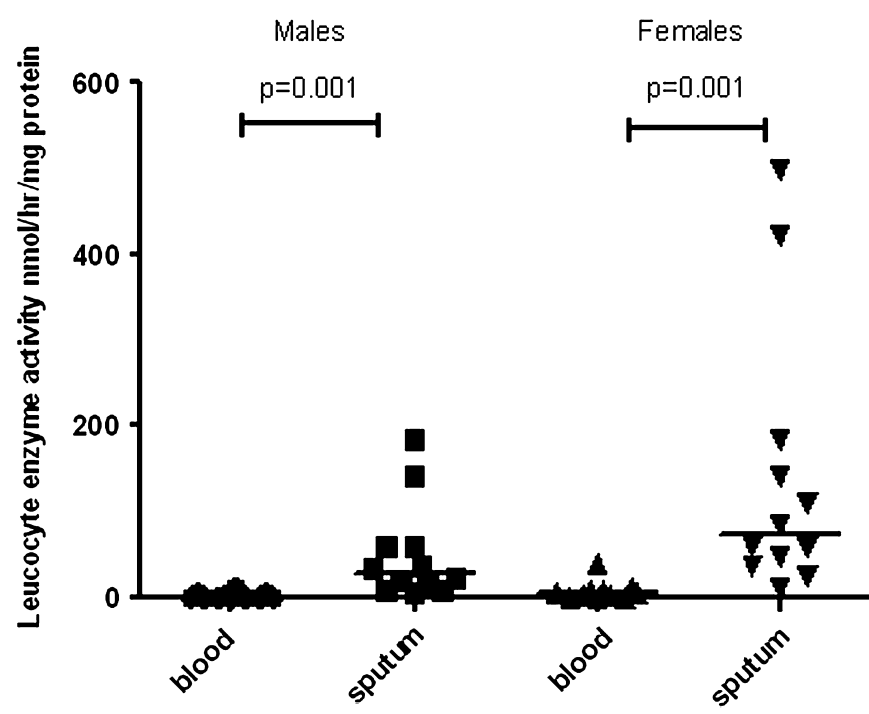

Abstract P179 Figure 1 Comparison of paired blood and induced sputum leucocyte enzyme activity in AFD males and females. 
GLA activity intracellularly in IS than peripheral blood, $\mathrm{p}=0.001$ (see Abstract P179 Figure 1), and extracellularly in IS supernatant versus plasma in males alone $(p<0.05)$.

Conclusions Sputum GLA activity is lower in male AFD patients than controls. However AFD subjects had significantly higher GLA activity in lung than blood. Measured airway obstruction in AFD was mild, though common. We speculate that the higher levels of lung enzyme found in AFD patients, on or off enzyme replacement, may contribute to the relative preservation of pulmonary function compared to other organ systems.

\section{P180 SPUTUM EOSINOPHIL POSITIVITY TO TAILOR STEROID MANAGEMENT OF SEVERE ASTHMATICS}

doi:10.1136/thx.2010.151043.31

G Tavernier, C Pris-Picard, R Gore, R Niven. University of Manchester, Wythenshawe Hospital, Manchester, UK

The use of sputum eosinophil count in asthma clinics is rapidly expanding as it has been reported as being a useful indicator of the worsening of asthma symptoms and that its normalisation reduces asthma exacerbations and admissions. Without additional steroids, levels of sputum eosinophils have been shown to be highly variable in severe asthmatic patients. Furthermore, precise patient phenotyping is increasingly becoming important as our understanding of the physiopathology of severe asthma widens. We introduced sputum differential cell counting in our severe asthma clinic, with a view to first reducing sputum eosinophils below 3\% by augmenting anti-inflammatory therapy, and attempting steroid withdrawal once patients became sputum eosinophil negative (E-). To date, 264 patients have been investigated for sputum eosinophils, using induction with nebulised sodium chloride if necessary and suitable. This paper presents our yearly update of the anti-inflammatory (steroid) therapy of the first successive patients with at least two successful sputum counts (current $n=71$ ), specifically investigating patients' management in the light of their positive sputum eosinophil levels at baseline assessment. Twenty patients were sputum eosinophil positive $(E+)$ on their initial visit and 25 had reduced eosinophil levels $(p=0.001)$ on a subsequent visit, including 14 becoming $\mathrm{E}-$. Nineteen were offered a trial of steroid augmentation: 11 patients with a trial of IM triamcinolone (all patients had subsequent reduced eosinophils levels, 9 becoming $\mathrm{E}-$, $\mathrm{p}=0.003)$;

5 patients with increased oral prednisolone treatment (four patients with reduced eosinophils levels, one becoming E-);

3 with increased inhaled steroid therapy (all with reduced eosinophil levels, 1 becoming $\mathrm{E}-$ ).

$66 \%$ of patients with uncontrolled sputum eosinophilia were treated with an increase in anti-inflammatory maintenance therapy. Sputum eosinophil levels decreased for $95 \%$ of these as already reported, but only 11/19 achieved full control of sputum eosinophilia with $2 / 11$ failing to normalise eosinophils despite IM triamcinolone (representing a population of confirmed steroid resistance. Sputum eosinophil negativity used as a surrogate marker for asthma control has been shown to be an essential tool in identification and management of patients with asthma at risk of deterioration and admission

\section{P181 BETTER ASTHMA CONTROL WITH MONTELUKAST THAN SALMETEROL IN ARG-16 HOMOZYGOUS CHILDREN WITH ASTHMA}

doi:10.1136/thx.2010.151043.32

${ }^{1} \mathrm{~K}$ Basu, ${ }^{2} \mathrm{H}$ P Donald, ${ }^{3} \mathrm{~B} J$ Lipworth, ${ }^{4} \mathrm{R}$ Tavendale, ${ }^{2} \mathrm{D}$ F Macgregor, ${ }^{5} \mathrm{~S}$ A Ogston, ${ }^{4} \mathrm{C}$ N A Palmer, 'S Mukhopadhyay. ${ }^{1}$ Royal Alexandra Children's Hospital, Brighton and
Sussex Medical School, Brighton, UK; ${ }^{2}$ Paediatric Asthma and Allergy Research Group, NHS Tayside, Perth, UK; ${ }^{3}$ Asthma and Allergy Research Group, Division of Medicine and Therapeutics, Ninewells Hospital, Dundee, UK; ${ }^{4}$ Population Pharmacogenetics Group, Biomedical Research Institute, University of Dundee, Dundee, UK; ${ }^{5}$ Division of Clinical and Population Sciences and Education, University of Dundee, Dundee, UK

Introduction and Objectives Diminished efficacy of salmeterol for improving asthma control is increased in children with asthma homozygous for arginine-16 (Arg16) allele of the ADRB2. Concerns have been raised regarding the efficacy and safety of long-term salmeterol use in patients with asthma. We investigated whether there is a genotype-specific difference in long-term asthma control with montelukast compared to salmeterol in individuals homozygous for $\operatorname{Arg} 16$ of $A D R B 2$

Methods In this pragmatic randomised controlled trial, 62 children (5-18 years) with asthma, carrying Arg/Arg16 genotype and exacerbation of asthma at least once within the previous year, were randomly assigned to receive Flixotide ${ }^{\circledR}$ (fluticasone propionate) via accuhaler (Diskus) dry powder inhaler device plus oral montelukast (Group I); or Seretide ${ }^{\circledR}$ (salmeterol plus equivalent dose of fluticasone) via accuhaler dry powder inhaler device plus placebo for montelukast (Group II). No effort was made to blind the prescribed inhaler. The primary end point was school absence, prospectively measured as individual events over the period of 1 year.

Results No significant difference was observed in school absences $(p=0.097)$ between the treatment groups. The use of reliever medication was significantly decreased in Group I compared to Group II $(p=0.004)$. Total exacerbations were reduced in Group 1 compared to Group $2(p=0.049)$. Self-reported symptoms were significantly improved in Group I compared to Group II (morning cough $\mathrm{p}=0.018$; morning wheeze $\mathrm{p}=0.001$; morning dyspnoea $p=0.008$; night wheeze: $p=0.004$; night dyspnoea: $p=0.001)$. A significant improvement in quality of life as per the Juniper paediatric asthma quality of life questionnaire was observed in Group I compared to Group II (activity limitation score $(\mathrm{p}=0.004)$, symptom score $(p=0.009)$, emotional function score $(p=0.002))$.

Conclusion In individuals homozygous for Arg16 of the ADRB2 locus, montelukast is an effective step up medication compared with salmeterol. Montelukast, as an asthma controller added on to inhaled steroid, improved asthma symptoms and quality of life, while reducing the use of reliever medication, in comparison to salmeterol. A larger randomised controlled trial is required, comparing asthma control with salmeterol versus montelukast in the genotypic subgroups in $A D R B 2$, and to explore the cost-effectiveness of genotypespecific controller therapies in children with asthma.

\section{P182 ADENOSINE POTENTIATES HUMAN MAST CELL FIBRINOLYTIC ACTIVITY}

doi:10.1136/thx.2010.151043.33

${ }^{1} \mathrm{M}$ J Sereda, ${ }^{2} \mathrm{P}$ Bradding, ${ }^{1} \mathrm{C}$ Vial. ${ }^{1}$ Department of Cell Physiology and Pharmacology, University of Leicester, ${ }^{2}$ Department of Infection, Immunity and Inflammation, University of Leicester

We investigated whether adenosine, a potent contributor to the regulation of pulmonary function, can modulate human lung mast cell (HLMC) fibrinolytic activity. Tissue plasminogen activator (tPA) activity and tPA transcript expression levels from a human mast cell line (HMC-1) and HLMC were monitored following adenosine application. Adenosine potentiated mast cell tPA activity and tPA gene expression in a dose-dependent manner. Adenosine effects were abolished in the presence of adenosine deaminase. HMC-1 cells predominantly expressed adenosine $A_{2 A}$ and $A_{2 B}$ receptor transcripts $\left(A_{2 A}>>A_{2 B}>A_{3}>>A_{1}\right)$. In addition to $A_{2 A}$ and $A_{2 B}, A_{3}$ receptor transcripts were also abundantly found in HLMC $\left(A_{2 A}>>A_{2 B}>>A_{3}>>A_{1}\right)$. Pharmacological and signalling studies 